\title{
Mitteilungen über veränderliche Sterne.
}

86. I9 I L Lrae near $\mu$ Lyrae.

On the plate R 73, I9I2 May I6 I I $40^{\mathrm{m}}$ Gr. m.t., exposure $\mathrm{I}^{\mathrm{b}} \mathrm{IO}^{\mathrm{m}}$, the variable or nova 86.19 I I Lyrae is the brighter of a pair of faint stars. At observed maximum I9 I I Oct. I it was brighter than $a$ (see the sketch in A. N. 4544), which is visually of $10^{\mathrm{m}}$. The variable is now however invisible with the 15 inch. reflector and is certainly $<4^{\mathrm{m}}$ visually and $<15^{\mathrm{m}}$ photographically. The faint companion is visible on three plates taken since I9I2 April Io.

Tatsfield, I 9 I 2 June I 8.

C. R. D'Esterre

\section{Var. 26.r9ז2 Geminorum.}

Nell' A. N. 4578 il Dr. $A$. $K i$ ihl pubblica una serie di osservazioni fatte sulla stella $\mathrm{BD}+33^{\circ} \mathrm{1433}$. Osservo che la media di queste grandezze è 6.87 e differisce di ${ }^{\mathrm{m}} \cdot 7$ dalla grandezza $6{ }^{\mathrm{m}} 8$ attribuita dalla PD alla stella $\mathrm{BD}$ $+33^{\circ}$ I 433, mentre tanto la grandezza media quanto le grandezze ottenute nelle stesse sere e attribuite dal Dr. Kühl alla $\mathrm{BD}+33^{\circ} \mathrm{I} 433$ e da me alla $\mathrm{BD}+32^{\circ} 1433$ vanno molto d'accordo, come risulta dal seguente specchietto:

\begin{tabular}{|c|c|c|}
\hline 1912 & $\begin{array}{c}\text { Gr. } \\
+32^{\circ} \mathrm{I} 433 \\
\text { Padova }\end{array}$ & $\begin{array}{c}\text { Gr. } \\
+33^{\circ} \text { I } 433 \\
\text { Kühl }\end{array}$ \\
\hline Marz.26 & $6^{\mathrm{m}} \cdot 94$ & - \\
\hline 28 & 7.03 & - \\
\hline $3^{\circ}$ & 6.82 & $6^{\mathrm{m}} \cdot 89$ \\
\hline Apr. $\quad 2$ & 6.90 & - \\
\hline 3 & 6.73 & 6.85 \\
\hline 4 & 6.88 & 6.89 \\
\hline 6 & 6.83 & - \\
\hline 7 & 6.68 & - \\
\hline
\end{tabular}

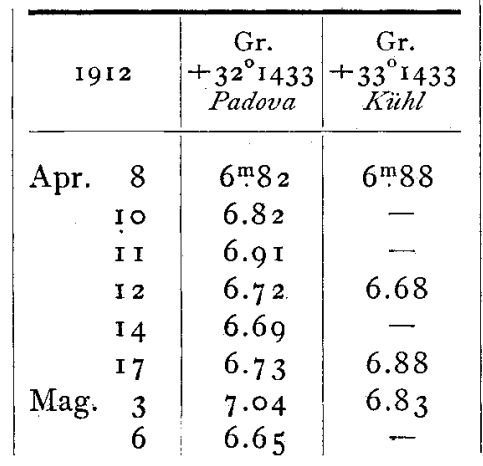

\begin{tabular}{|r|c|c|}
\hline I912 & $\begin{array}{c}\mathrm{Gr} . \\
+32^{\circ} \text { 14333 } \\
\text { Padova }\end{array}$ & $\begin{array}{c}\text { Gr. } \\
+33^{\circ} \text { I } 433 \\
\text { Kiihl }\end{array}$ \\
\hline Mag. 9 & 6.66 & - \\
I I & 6.70 & - \\
I 2 & 6.80 & $6^{\mathrm{m}} 80$ \\
I3 & 6.52 & -
\end{tabular}

\begin{tabular}{|c|c|c|}
\hline 1912 & $\begin{array}{c}\mathrm{Gr} . \\
+3^{\circ} \mathbf{1} 433 \\
\text { Padova }\end{array}$ & $\begin{array}{c}\text { Gr. } \\
+33^{\circ} \text { I } 433 \\
\text { Kii hl }\end{array}$ \\
\hline Mag. I 4 & $6^{\mathrm{m}} \cdot 90$ & - \\
\hline I 7 & 6.80 & - \\
\hline т 8 & $6.6 \mathrm{I}$ & - \\
\hline I 9 & 6.80 & - \\
\hline
\end{tabular}

Grandezza media: Padova $6^{\mathrm{m}} \cdot 79$, Kühl 6m.87.

R. Osservatorio Astronomico, Padova, ig r 2 Giugno 19.

Dr. E. Padova.

Der von Herrn Dr. Padova gegen meine Helligkeitsangaben für $\mathrm{BD}+33^{\circ}$ I 433 erhobene Einwand läßt sich sofort entkräften durch den nochmaligen Hinweis darauf, daß meine Zahlen die photographische Helligkeit angeben eine Verwechslung mit $\mathrm{BD}+32^{\circ}{ }^{\circ} 433$ ist, wie ich mich aufs neue überzeugt habe, völlig ausgeschlossen. Wie in A.N. 4586 näher auseinandergesetzt, habe ich meine Vergleichsterne an die photographischen Größen der Präsepesterne nach Schwarzschild (Publ. Wien-Ottakring V, C', Kap. VI) angeschlossen. Die Differenz $m_{\text {phot. }}$ - Potsd. $=0^{\mathrm{m}} \cdot{ }^{\mathrm{I}} \mathrm{I}$, wie sie $\mathrm{BD}+33^{\circ} \mathrm{I} 433$ zeigt, ist bei dem Spektraltypus F 8 durchaus nicht auffällig und im Einklang mit den von Schwarzschild a. a. O. S. 98 angeführten Zahlen.

Die von Herrn Dr. Padova an $\mathrm{BD}+32^{\circ} 1433$ bemerkten Lichtschwankungen von etwa $\circ^{\mathrm{m}} \cdot 4$ fehlen übrigens in meinen photographischen Helligkeitszahlen völlig. Dieser Stern zeigt bei mir (A. N. 4586) in den einzelnen Abendwerten von 16 Beobachtungstagen gegen das Mittel Abweichungen, die einmal $\circ^{\mathrm{m}} .{ }_{7}$ und sonst stets weniger als $\circ$ mo5 betragen.

München, r9 I 2 Juli I I.

A. Kühl.

Anzeige. Der Raum der Astronomischen Nachrichten wurde in letzter Zeit durch die unmittelbaren wissenschaftlichen Aufgaben derart in Anspruch genommen, daß es nicht möglich war, die Zeitschrift daneben in dem wünschenswerten Maße den literarischen Interessen der Leser dienstbar zu machen. Um diesem Zwecke nun in der Folge wieder in wirksamer Weise dienen zu können, will der Herausgeber den Versuch machen, der Zeitschrift ein regelmäßig erscheinendes Beiblatt anzugliedern, in welches die bisher im Hauptblatte veröffentlichten literarischen Anzeigen aufgenommen werden sollen. Außerdem sollen im Beiblatte Referate über an anderer Stelle veröffentlichte wichtige Arbeiten gegeben und insbesondere über den Inhalt verwandter Zeitschriften berichtet werden. Endlich soll das Beiblatt Anzeigen literarischen und technischen Inhaltes aus den Kreisen des Buchhandels und der Technik den Lesern zur Kenntnis bringen, eine Aufgabe, der die Zeitschrift in den ersten Jahrzehnten ihres Erscheinens sich gleichfalls vielfach gewidmet hat.

Mit vorliegender Nummer kommt das Beiblatt zum ersten Male zur Ausgabe und gestatte ich mir, dasselbe dem Interesse und der eifrigen Förderung der Leser der Astronomischen Nachrichten angelegentlichst zıl emptehlen.

Kiel, 19 I 2 Juli 30.

Hermann Kobold.

Druckfehler. Im Katalog von I0663 Sternen für das Äquinoktium r 900 (Bonner Veröffentlichungen Nr. Io) lies pag. 227 bei Nr. $545^{8}$ Deklination $+49^{\circ} 32^{\prime} 20^{\prime \prime} 80$ statt $+49^{\circ} 32^{\prime} 29^{\prime \prime} 80$. F. Kiistner.

Berichtigung zu Nr. 4574 Bd. $19 \mathrm{r}$ p. 267 Z. 22 v. u. April 16 10 ${ }^{\text {h }} 23^{\mathrm{m}}$ statt: $c 3 N$ lies: $N 3 c$.

Inhalt zu Nr. 4593. F. E. Ross. The Kimura term in the Latitude Variation and the Constant of Aberration. I33. - W. H. Pickering. Hyperbolic Comets. I4I. - Sextantenbeobachtungen des Kometen 19 I I c (Brooks). 143. - Mitteilungen über kleine Planeten. 145. - Mitteilungen über veränderliche Sterne. 147. - Anzeige. 147. - Druckfehler. I47. - Berichtigung. 147 .

Geschlossen Igrz Aug 2. Herausgeber: H. Kobold. Druck von C. Schaidt. Expedition : Kiel, Moltkestr. 80. 